\title{
Nursing Study on Reducing the Incidence of Bleeding and Exudation after PICC Catheterization by Blunt Separation Combined with Early Sheath Removal
}

\author{
Lili Tian*, Lin Ye \\ Zhenjiang First People's Hospital, Zhenjiang 212002, Jiangsu Province, China \\ *Corresponding author: Lili Tian, 75089068@qq.com
}

\begin{abstract}
Objective: To compare the effects of blunt separation combined with early sheath removal and conventional catheterization on the incidence of bleeding, exudation and subcutaneous congestion after peripherally inserted central catheter (PICC). Methods: 250 patients with PICC catheterization in our hospital were selected, including 125 in the control group and 125 in the observation group. The conventional catheterization method was used in the control group: The sheath was removed by scalpel skin expansion and complete tube delivery in place. The observation group used blunt separation combined with early sheath removal. The success rate of one-time sheath delivery, immediate bleeding, 24-hour bleeding and exudation were compared. Results: The success rate of sheath delivery in the observation group was 100\%; The amount of immediate blood loss and blood loss $24 \mathrm{~h}$ after catheterization in observation group was obviously lower than that in the control group, with statistical significance $(\mathrm{P}<0.05)$. There were 6 cases of exudation in the control group and no exudation in the observation group. There was significant difference in the incidence between the two groups $(\mathrm{P}<0.05)$; There were 10 cases of subcutaneous congestion in the control group and 2 cases of subcutaneous congestion in the observation group. There was significant difference in the incidence between the two groups $(\mathrm{P}<0.05)$; Conclusion: Blunt separation combined with early sheath removal can reduce the occurrence of local blood and fluid leakage after PICC catheterization.
\end{abstract}

Keywords: Blunt separation; Early sheath removal; PICC; Bleeding and exudation; Subcutaneous congestion

Publication date: November 2021; Online publication: November 30, 2021

\section{Introduction}

The PICC catheter placement on the elbow under the guidance of ultrasound combined with Modified Seldinger Technique (MST) is widely used in clinical practice. Compared with the traditional puncture and catheterization method under the elbow, it improves the success rate of catheterization, reduces the incidence of complications such as phlebitis and thrombus, and it is more comfortable to move at the same time. The hospital is a Level 3 Class A General Hospital. PICC catheterization under ultrasound guidance combined with MST has been adopted since 2011. During catheterization and maintenance, it was found that the incidence of bleeding and exudation from elbow catheterization was also increasing, which may be related to skin expansion by scalpel and long residence time of vagina vasorum in blood vessels. The literature reported that the incidence of local bleeding within 24 hours was more than $80 \%$ [1-3], and even $100 \%$ in patients with bleeding tendency ${ }^{[4]}$. Shentu Yingqin et al. ${ }^{[5]}$ reported that the incidence of bleeding was $2.21 \%$, and Leung et al. ${ }^{[6]}$ reported that the incidence of continuous bleeding and exudation (> 3d) at the puncture point was $24.1 \%$. It has been studied in the literature that the improved sheath delivery method can reduce the occurrence of bleeding and exudation from PICC catheterization ${ }^{[7]}$. In this study, the 
procedure of sheath delivery was improved. First, the blunt separation method combined with scalpel was used to replace the skin expansion by scalpel, so as to avoid the damage of sharp instruments to subcutaneous tissues, blood vessels and lymphatic vessels; Second, the sheath was removed when the tube is delivered to $15 \mathrm{~cm}$, so as to reduce the residence time of the vagina vasorum in the blood vessel, reduce the damage of the vascular intima, and reduce the occurrence of blood seepage, which has received good results and are reported as follows.

\section{Materials and methods}

\subsection{General information}

Patients who underwent PICC catheter chemotherapy in the intravenous infusion nursing center of our hospital from June to December 2020 were selected. Inclusion criteria: age $\geq 16$ years; Signed informed consent; Blood routine and coagulation function are normal. Exclusion criteria: restless and uncooperative patients; Patients with puncture failure or difficult delivery during catheterization. 250 cases were included and divided into observation group and control group according to random number table method and catheterization order, 125 cases in each group. There were 65 males and 60 females in the observation group, aged from 20 to 83 years. They were 79 cases of right basilic vein catheterization, 32 cases of left basilic vein catheterization, 11 cases of right brachial vein catheterization and 3 cases of left brachial vein catheterization. There were 62 males and 63 females in the control group, aged from 23 to 81 years. They were 83 cases of right basilic vein catheterization, 28 cases of left basilic vein catheterization, 7 cases of right brachial vein catheterization and 7 cases of left brachial vein catheterization. The selected vascular catheterization depth was $\leq 1.5 \mathrm{~cm}$. There was no significant difference in gender, age, puncture vessels and types of diseases between the two groups $(\mathrm{P}>0.05)$.

\section{Methods}

\subsection{General materials}

The catheters used in this study are 4Fr three-way valve type single lumen PICC tube produced by American Bard company, STTE RITE 5 ultrasound instrument, MST vagina vasorum kit, special puncture bag for PICC catheterization and special drug change bag (uniformly configured by our hospital).

\subsection{Methods}

The catheterization was completed by a full-time nurse of intravenous infusion nursing specialty in Jiangsu Province. The PICC catheterization process was carried out under the guidance of ultrasound combined with MST technology in accordance with the relevant PICC operating procedures of INS in the United States, Nursing Practice Guidelines and Implementation Rules for Infusion Therapy and Technical Code of Operation for Venous Therapy and Nursing in China. The catheterization sites were above the elbow. Routine skin disinfection was performed, maximum sterile barrier was established, and local anesthesia was performed. Ultrasound-guided puncture combined with MST technology was successful, and guide wire was sent into the body, and about $10 \mathrm{~cm}$ guide wire was retained outside the body. The control group was treated with longitudinal skin expansion: The scaler knife was held, the back of the scaler knife was inserted on the guide wire, the blade was vertical to the skin, and the tip of the knife was inserted into the skin lengthways to expanding the skin, so that the blade enters $1 / 3$. The puncture sheath was pushed subcutaneously through the guide wire and along the guide wire into the vessel subcutaneously, the guide wire and inner sheath was removed, the catheter to the reserved scale was slowly fed, and the outer sheath was removed. Ultrasonography was used to preliminarily confirm the abnormal position of the catheter, trim the catheter, and install decompression sleeve as well as heparin cap, positive pressure sealing tube, fixed catheter, X-ray location and activity guidance. The observation group was treated with blunt 
separation combined with early sheath withdrawal: Without using a scalpel, the inner and outer sheath of the vagina vasorum was separated first, and then the outer sheath of the $14 \mathrm{G}$ traditional puncture needle supporting the catheter was separated from the steel needle. Then the outer sheath of the traditional puncture needle was covered outside the inner sheath of the vagina vasorum, the skin was tightened, the component was pushed subcutaneously through the guide wire and along the guide wire into the blood vessel, the guide wire and the inner sheath was removed and slowly fed into the catheter. When the catheter was inserted about $15 \mathrm{~cm}$, the catheter was immediately withdrawn from the outer sheath under the premise that the catheter was not pulled out, and the catheter was inserted to the predicted length. The other steps were the same as the control group. After catheterization, a 4-layer $5 * 5 \mathrm{~cm}$ sterile gauze was folded in half (8 layers) and pressed above the puncture point, and a $10 * 12 \mathrm{~cm}$ transparent application was pasted outside. The elastic bandage was wrapped around the arm three times to assist in pressing the puncture point, and the tightness should be 2 fingers. The next day, the full-time nurse of the placement center made a return visit, evaluation and dressing change at the bedside.

\subsection{Evaluation index}

(1) Success rate of one-time sheath delivery: The success rate of sheath delivery in the control group was used. If skin expansion was needed, it was regarded as unsuccessful.

(2) Count the number of cases of immediate bleeding and 24-hour bleeding: The small amount of bleeding is that the wetted area is less than or equal to $1 / 2$ gauze; The medium amount of bleeding is that the wetted area is greater than $1 / 2$ and less than 1 gauze; The large amount of bleeding is soaking 1 or more gauze ${ }^{[8]}$.

(3) Number of cases of exudation at puncture point.

(4) Increased maintenance times and cases from 24 hours to one week cycle maintenance.

(5) The number of cases of subcutaneous congestion after one week.

\subsection{Statistical methods}

The data were collected in tables, and the units were expressed in cases and percentages. SPSS23.0 software was used for statistical analysis; chi-square test and rank sum test were used to test the level $a=0.05$.

\section{Results}

Table 1. The success rate of one-time skin expansion

\begin{tabular}{|c|c|c|c|c|c|c|c|c|c|c|c|c|c|}
\hline \multirow{2}{*}{ Group } & \multirow{2}{*}{$\begin{array}{l}\text { Number } \\
\text { of cases }\end{array}$} & \multirow{2}{*}{$\begin{array}{c}\text { Success rate of } \\
\text { one-time skin } \\
\text { expansion }\end{array}$} & \multicolumn{3}{|c|}{ Immediate bleeding volume } & \multicolumn{3}{|c|}{ 24h bleeding volume } & \multicolumn{3}{|c|}{$\begin{array}{l}\text { Increased maintenance } \\
\text { times within } 7 \mathrm{~d}\end{array}$} & \multirow{2}{*}{ 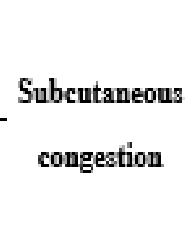 } & \multirow{2}{*}{$\begin{array}{c}\text { Puncture } \\
\text { point } \\
\text { exudation }\end{array}$} \\
\hline & & & $\begin{array}{c}\text { Small } \\
\text { amount }\end{array}$ & $\begin{array}{l}\text { Medium } \\
\text { amount }\end{array}$ & $\begin{array}{l}\text { Large } \\
\text { amount }\end{array}$ & $\begin{array}{l}\text { Small } \\
\text { amount }\end{array}$ & $\begin{array}{r}\text { Medium } \\
\text { amount }\end{array}$ & $\begin{array}{l}\text { Large } \\
\text { amount }\end{array}$ & 1 time & 2 times & 3 times & & \\
\hline Control group & 125 & $100 \%$ & 69 & 51 & 5 & 106 & 17 & 2 & 45 & 5 & 1 & 10 & 6 \\
\hline $\begin{array}{c}\text { Observation } \\
\text { group }\end{array}$ & 125 & $100 \%$ & 100 & 24 & 1 & 120 & 5 & 0 & 20 & 1 & 0 & 2 & 0 \\
\hline$P$ value & & & & 0 & & & 0.003 & & & 0 & & 0.038 & 0.039 \\
\hline $\mathrm{X}^{2}$ value $/ \mathrm{Z}$ & & & & $\mathrm{Z}=4.233$ & & & $Z=3.016$ & & & $Z=6.729$ & & 4.289 & 4.269 \\
\hline
\end{tabular}




\section{Discussion}

\subsection{Effectively reduce bleeding}

During PICC catheterization under ultrasound guidance combined with MST, because the blood vessels are mostly $0.5 \sim 2.5 \mathrm{~cm}$ deep under the skin, the puncture needle, vascular sheath and catheter travel a long distance under the skin, which is easy to cause subcutaneous tissue damage, and there is bleeding and exudation at the puncture site after catheterization ${ }^{[9]}$. At the same time, the scalpel is used to expand the skin in the process of catheterization, which destroys the dermis of the skin, easily damages the blood vessels, lymphatic vessels and nerves in the dermis, and also causes bleeding and exudation at the puncture point ${ }^{[10]}$. Although it is reported in the literature that longitudinal skin expansion ${ }^{[11]}$ can reduce the occurrence of bleeding and exudation, it may still cause damage to subcutaneous tissue, capillaries and lymphatic capillaries. During the process of bleeding and compression at the puncture point, the blood will penetrate into the subcutaneous tissue and cause subcutaneous congestion. Based on the problems existing in the above operations, this study improved the sheath-feeding process in the pipe-placing process. Blunt separation method can effectively reduce the damage to subcutaneous tissues and blood vessels, and has no significant influence on the success rate of skin expansion. At the same time, combined with the early withdrawal of the sheath, the residence time of the vascular sheath is shortened, and the stimulation of the vascular intima by foreign bodies is reduced. For beginners, it is difficult to master the position and strength of vascular compression. Too much pressure will increase the damage to the intima of the vascular sheath, the amount of blood loss in the vascular sheath will be relatively large, and it is not easy to observe the amount of blood loss. It is easy to master the pressure after early removal of the sheath, so as to reduce the amount of blood loss and make the puncture site heal earlier. The results show that it can effectively reduce the occurrence of bleeding: The amount of immediate bleeding, the amount of bleeding in 24 hours and the increased maintenance times in one week were significantly different from those in the control group $(\mathrm{P}<$ $0.01)$, and the subcutaneous congestion was also statistically significant compared with the control group $(\mathrm{P}<0.05)$.

\subsection{Reduce the occurrence of exudation}

In case of exudation at the PICC puncture point, there is no particularly effective treatment method, which can only promote healing by increasing the dressing change frequency and pressing the puncture point, and it is easy to develop into local infection, phlebitis, even blood flow infection and thrombosis, with serious consequences. In this study, there was no exudation at the puncture point in the observation group and 6 cases in the control group, which showed that blunt separation could reduce the occurrence of exudation at the puncture point $(\mathrm{P}<0.05)$.

\subsection{Disadvantage}

There are several puzzles in this study. First, patients with vascular depth are the weak link of this study, because the outer sheath of traditional puncture needle is only $3.2 \mathrm{~cm}$, and the length entering the blood vessel is short. When the vascular depth is $\geq 2.5 \mathrm{~cm}$, once the patient is slightly agitated, it is likely to fail to send the sheath. Second, the blunt separation method is only used in 4Fr three-way valvular single lumen PICC tube, and other types of catheters have not been implemented. Third, if blood vessels are prone to spasm, early sheath removal may cause tube delivery failure. The above points need to be verified with a larger sample size in the future nursing work, so as to be further optimized and improved.

\section{Disclosure statement}

The authors declare no conflict of interest. 


\section{References}

[1] Zhang LL, Liu JH, 2013, Observation of Exudation at Postoperative Puncture Site in 185 Patients with PICC Catheterization. Jiangsu Health Care, 15(6): 26-27.

[2] Wang X, Wang XY, 2012, Cause Analysis and Countermeasures of Local Bleeding after PICC Catheterization. Today Nurse, (2): 158-159.

[3] He XY, 2011, Application of Early Nursing Intervention in Reducing Bleeding at P1CC Puncture Sites. Chinese Journal of Misdiagnostics, 11(11): 2582.

[4] Li Y, Zhang JF, Zhang H, 2016, Cause Analysis and Nursing Research Progress of Continuous Bleeding after PICC Catheterization in Patients with Leukemia Journal of Nurses Training, 31(4): 319 321.

[5] Shentu YQ, Zhang RY, Chen CF, 2011, Cause Analysis and Nursing Countermeasures of 27 Cases of Fluid Exudation at PICC Puncture Site. Chinese Journal of Nursing, 46(2): 131-132.

[6] Leung TK, Lee CM, Tai CJ, et a1., 2011, A Retrospective Study on the Long-Term Placement of Peripherally Inserted Central Catheters and the Importance of Nursing Care and Education. Cancer Nursing, 34(1): 25-30.

[7] Zhao LF, Cao CZ, Wang YP, et al., 2017, Improved Scabbard Delivery method to Reduce the Incidence of PICC-related Exudation. Nursing and Rehabilitation Journal, 3(16): 203-205.

[8] Wang J, Zeng L, 2014, Influence of Two Kinds of Catheter Sheath Insertion Methods on Hemorrhage of PICC with Modified Sedinger Technology. Journal of Nursing, 19(7): 60 - 62.

[9] Tong J, Feng LJ, Han XH, et al., 2013, Analysis and Nursing of the Causes of Effusion at the Puncture Point of PICC Catheterization with Improved Sedinger Technology under the Guidance of Ultrasound. Journal of Nursing Science, 28(21): 46-47.

[10] Chen LJ, Zhu W, Qin XY, et al., 2018, Evaluation of Two Different Blunt Separation Methods for Central Venous Catheterization Through Ultrasound-guided Peripheral Venipuncture. Nursing Journal of Chinese People's Liberation Army, 35(24): 56-58.

[11] Xu Y, Li RM, Fu R, 2014, Comparison of PICC Tube Expansion Angle with Modified Seidinger Technique under Ultrasonic Guidance. Journal of Nursing Science, 29(1): 40-41. 